\title{
PARTICIPACIÓN Y NUEVA GESTIÓN PÚBLICA. UN ANÁLISIS MUNICIPAL TUNJA, DUITAMA Y SOGAMOSO
}

Laura Margarita Barreto Bernal Jaime Ignacio Bermúdez Guerrero Patricia Carolina Barreto

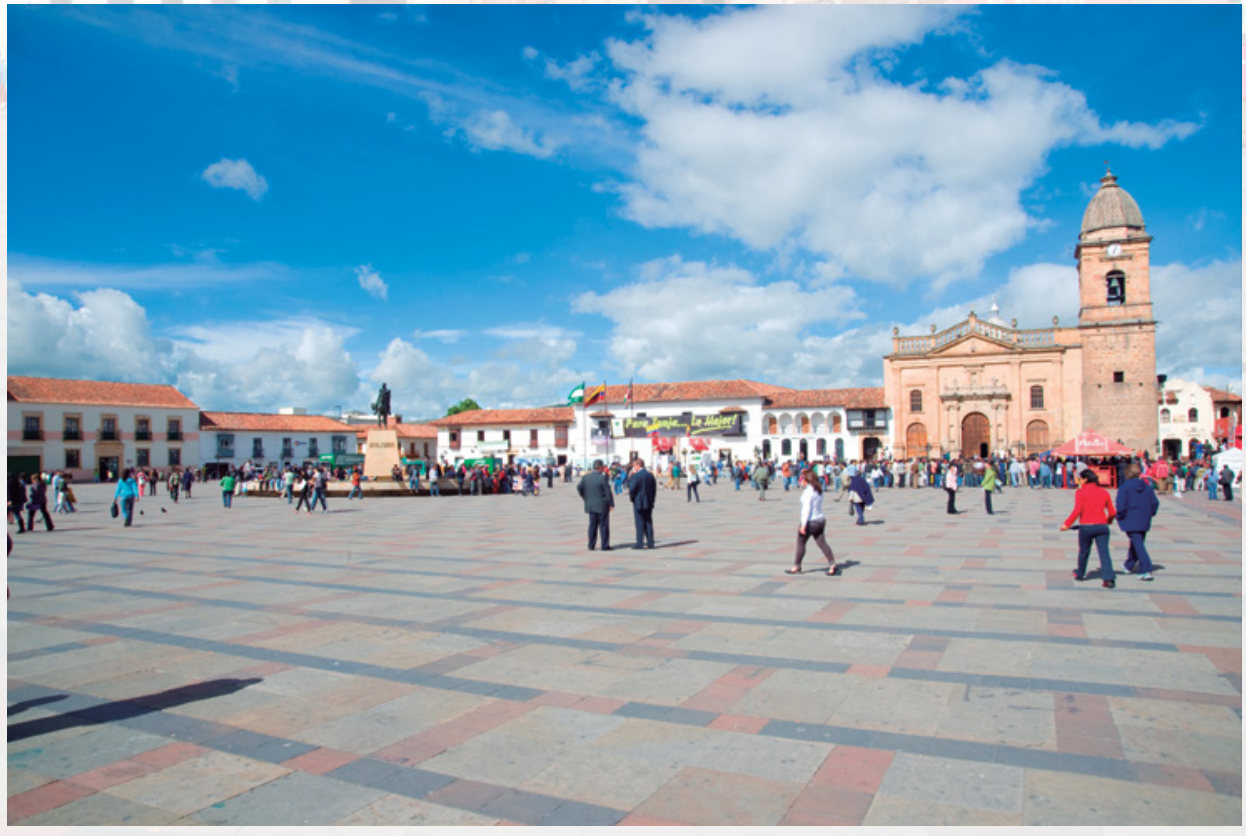





\title{
PARTICIPACIÓN Y NUEVA GESTIÓN PÚBLICA. UN ANÁLISIS MUNICIPAL TUNJA, DUITAMA Y SOGAMOSO*
}

\author{
Laura Margarita Barreto Bernal \\ Jaime IgnaCio Bermúdez Guerrero \\ Patricia Carolina Barreto \\ Universidad Antonio Nariño \\ Universidad Pedagógica y Tecnológica de Colombia
}

\section{Resumen}

El presente artículo es resultado de una investigación realizado por la Universidad Antonio Nariño, la Universidad Pedagógica y Tecnológica de Colombia y la Universidad Nacional Abierta a Distancia (UNAD) denominada "Evidencia pragmática de los elementos centrales de la nueva gestión pública (NGP) en tres municipios de Boyacá: Tunja, Duitama y Sogamoso, período 2012-2015”. Mediante un análisis documental se examinaron los datos obtenidos sobre participación ciudadana. Se expone una contextualización conceptual sobre la NGP y la participación ciudadana como fuente de legitimidad y, más adelante, se presentan la metodología empleada, los resultados y las conclusiones. Se observó un uso diferenciado de los espacios de participación y se identificó su uso para la construcción de legitimidad de manera preponderante en las primeras etapas del ciclo de gestión.

Palabras clave: participación ciudadana, nueva gestión pública, legitimidad, ciclo de gestión, políticas públicas, participación local.

Resultado de la investigación titulada: "Evidencia pragmática de los elementos centrales de la nueva gestión pública en tres municipios de Boyacá: Tunja, Duitama y Sogamoso, período 2012-2015”, realizada por el grupo de investigación Poder, de la Universidad Pedagógica y Tecnológica de Colombia, en conjunto con la Universidad Antonio Nariño y la Universidad Nacional Abierta y a Distancia (UNAD). Finalizó en julio de 2019. 
Los autores: Laura Margarita Barreto Bernal, magíster en Políticas Públicas. Docente investigadora y coordinadora del Programa de asistencia legal a personas con necesidad de protección internacional del consultorio jurídico de la Universidad Antonio Nariño, sede Duitama. ORCID: https://orcid. org/0000-0003-4386-5023. Correo electrónico: laura.barreto@uan.edu.co

Jaime Ignacio Bermúdez Guerrero, especialista en Administración Pública. Docente de la Escuela de Administración de Empresas de la Universidad Pedagógica y Tecnológica de Colombia. ORCID: 0000-0001-8839-6860. Correo electrónico: jaime.bermudez@uptc.edu.co

Patricia Carolina Barreto, doctora en Administración. Docente investigadora de la Universidad Pedagógica y Tecnológica de Colombia. ORCID: 0000-0001-5349-9712. Correo electrónico: patricia. barreto@uptc.edu.co

Recibido: 15 de junio de 2021; evaluado: 29 de septiembre de 2021; aceptado: 14 de octubre de 2021. 


\title{
PARTICIPATION AND NEW PUBLIC MANAGEMENT: AN ANALYSIS AT THE MUNICIPAL LEVEL IN TUNJA, DUITAMA, AND SOGAMOSO
}

\author{
Laura Margarita Barreto Bernal \\ Jaime Ignacio Bermúdez Guerrero \\ Patricia Carolina Barreto \\ Universidad ANTONio Nariño \\ Universidad Pedagógica y Tecnológica de Colombia
}

\begin{abstract}
This article is the result of a research conducted by the Universidad Antonio Nariño, the Universidad Pedagógica y Tecnológica de Colombia, and the Universidad Nacional Abierta a Distancia (UNAD), called "Pragmatic evidence of the central elements of new public management (NPM) in three municipalities of Boyacá: Tunja, Duitama, and Sogamoso, in 2012-2015." The researchers examined data obtained on citizen participation through a documentary analysis within the framework of the administrations of the studied municipalities. A conceptual contextualization of NPM and citizen participation as a source of legitimacy is presented, followed by a description of the applied methodology, results, and conclusions. It was possible to evidence a differentiated use of participatory spaces; similarly, their use for the construction of legitimacy was identified in a predominant way in the early stages of the management cycle.
\end{abstract}


Keywords: citizen participation, new public management, legitimacy, management cycle, public policies, local participation.

About the authors: Laura Margarita Barreto Bernal, MA in Public Policy, research professor and coordinator of the legal assistance program for people in need of international protection at the legal office of the Universidad Antonio Nariño, Duitama campus. ORCID: https://orcid.org/0000-00034386-5023. E-mail: laura.barreto@uan.edu.co

Jaime Ignacio Bermúdez Guerrero, Specialization in Public Administration, professor at the School of Business Administration of the Universidad Pedagógica y Tecnológica de Colombia. ORCID: 0000-0001-8839-6860. E-mail: jaime.bermudez@uptc.edu.co

Patricia Carolina Barreto, PhD in Administration, research professor at the Universidad Pedagógica y Tecnológica de Colombia. ORCID: 0000-0001-5349-9712.E-mail: patricia.barreto@uptc.edu.co

Received: June 15, 2021; evaluated: September 29, 2021; accepted: October 14, 2021. 


\title{
PARTICIPAÇÃO CIDADÃ E NOVA GESTÃO PÚBLICA. UMA ANÁLISE MUNICIPAL DE TUNJA, DUITAMA E SOGAMOSO, COLÔMBIA
}

\author{
Laura Margarita Barreto Bernal \\ Jaime Ignacio Bermúdez Guerrero \\ Patricia Carolina Barreto \\ Universidad Antonio Nariño \\ Universidad Pedagógica y Tecnológica de Colombia
}

\section{Resumo}

Este artigo é resultado de uma pesquisa realizada pela Universidad Antonio Nariño, pela Universidad Pedagógica y Tecnológica de Colombia e pela Universidad Nacional Abierta a Distancia, denominada "Evidência pragmática dos elementos centrais da nova gestão pública em três municípios de Boyacá, Colômbia: Tunja, Duitama e Sogamoso, período 2012-2015". A partir de uma análise documental, foram examinados os dados obtidos sobre participação cidadã. É exposta uma contextualização conceitual sobre a nova gestão pública e a participação cidadã como fonte de legitimidade e, mais adiante, são apresentados a metodologia, os resultados e as conclusões. Foi observado um uso diferenciado dos espaços de participação e identificado seu uso para a construção de legitimidade de maneira preponderante nas primeiras etapas do ciclo de gestão.

Palavras-chave: participação cidadã, nova gestão pública, legitimidade, ciclo de gestão, políticas públicas, participação local. 
Os autores: Laura Margarita Barreto Bernal, mestra em Políticas Públicas, docente pesquisadora e coordenadora do programa de assistência legal a pessoas com necessidade de proteção internacional do consultório jurídico da Universidad Antonio Nariño, campus Duitama. ORCID: https://orcid. org/0000-0003-4386-5023.Correio eletrônico: laura.barreto@uan.edu.co

Jaime Ignacio Bermúdez Guerrero, especialista em Administração Pública, docente da Escuela de Administración de Empresas da Universidad Pedagógica y Tecnológica de Colombia. ORCID: 0000-0001-8839-6860. Correio eletrônico: jaime.bermudez@uptc.edu.co

Patricia Carolina Barreto, doutora em Administração, docente pesquisadora da Universidad Pedagógica y Tecnológica de Colombia. ORCID: 0000-0001-5349-9712. Correio eletrônico: patricia.barreto@ uptc.edu.co

Recebido: 15 de junho de 2021; avaliado: 29 de setembro de 2021; aceito: 14 de outubro de 2021. 


\section{Introducción}

El presente artículo tiene como objetivo establecer el papel de la participación en la construcción de legitimidad desde la perspectiva de la nueva gestión pública (NGP) como modelo de gestión identificado en la práctica administrativa colombiana, a partir del estudio comparativo de las gestiones realizadas entre los años 2012 y 2015 en los tres principales municipios del departamento de Boyacá. Se utilizó como referente metodológico el modelo abierto de gestión para resultados, elaborado por el Banco Interamericano para el Desarrollo (BID) y el Centro Latinoamericano de Administración para el Desarrollo (CLAD). ${ }^{1}$

El problema de investigación consiste en identificar si la participación ciudadana, como medio para crear legitimidad alrededor de la acción del Gobierno, es usada como un requisito formal y no como un elemento de fondo en la construcción de eficacia y eficiencia, criterios básicos de la NGP. Esta adopta la participación siempre que permita cumplir la misión del Gobierno, ayude a adaptarse a las circunstancias y posibilite generar valor para los ciudadanos, así que la exclusiva participación electoral obliga a la adopción de valores como la transparencia, la rendición de cuentas y la equidad.

Entre los inconvenientes que se debieron superar surgió la extracción de los datos de una investigación mayor que no solo evaluaba la participación en la gestión pública, sino también otros aspectos, de acuerdo con sus objetivos. Además, se hallaron obstáculos en la búsqueda de las fuentes de información, en especial en cuanto a soportes de los procesos de participación desarrollados en las Administraciones estudiadas, debido a dos factores: el primero, porque se indagó en vigencia de una Administración posterior a las analizadas y, segundo, por la dificultad general en el seguimiento de la memoria institucional, sobre todo, de las acciones de participación de las cuales pocas veces se deja registro, porque son consideradas tangenciales a las actividades administrativas.

A pesar de estas situaciones se encontraron datos suficientes para hacer una lectura objetiva y descriptiva de la situación en la que se desarrolla la participación en un contexto de nueva gestión pública como modelo de administración identificado en los municipios objeto de estudio. Se observó una participación tímida, en tanto su

Mauricio García y Roberto García López, "Modelo abierto de gestión para resultados en el sector público", Revista del CLAD Reforma y Democracia, núm. 39 (2007), https://www.redalyc.org/pdf/3575/357533693007. pdf (acceso septiembre 15, 2021). 
función de legitimación y concreción de los principios de la nueva gestión pública, lo que reafirma las críticas a este modelo.

El presente artículo comienza con una aproximación al papel de la participación en el modelo NGP; en segundo lugar, establece su relación con la función de legitimidad; en seguida, como tercer elemento, describe el instrumento utilizado y, por último, expone los resultados encontrados y las conclusiones.

\section{La participación ciudadana en la nueva gestión pública}

Una de las preocupaciones de la NGP es la creación de valor público en la provisión de bienes y servicios que los ciudadanos no pueden obtener en el sector privado. Una forma de incrementar dicho valor es involucrar el diálogo comunitario y la participación social ${ }^{2}$ en el proceso de producción de esos bienes y servicios. La NGP que aplica la lógica de la empresa privada a la Administración pública recurre a la participación para lograr un equilibrio.

Como contrapeso a la introducción de la lógica de mercado como orientador principal de las decisiones administrativas, la NGP propone el fortalecimiento de la participación de los ciudadanos mediante el desarrollo de estrategias tipo veedurías, comité de vigilancia o asociaciones de usuarios. También favorecerá la participación a los procesos de decisión, de seguimiento (monitoreo) y de ejecución de las diversas formas asociativas de la sociedad civil. ${ }^{3}$

El empleo de la NGP en los Gobiernos locales puede ser promovido en la puesta en marcha de espacios de participación ciudadana, siempre que la participación promovida apunte a: i) desarrollar la misión propuesta en el gobierno específico; ii) adaptar la Administración a las circunstancias cambiantes, y iii) aprovechar el espacio para crear más cosas buenas para los ciudadanos. ${ }^{4}$ Desconocer estos criterios no tendría utilidad para la ejecución de un Gobierno eficaz, eficiente y legítimo.

Entre las estrategias que la Organización para la Cooperación y Desarrollo Económico (OCDE) propuso para la implementación de la NGP estableció la de "proveer

2 José María Ramos y Marcela Reyes, "Gobiernos locales y participación ciudadana: hacia un enfoque de gestión estratégica asociada”, Espiral 12, núm. 34 (2005): 55, http://www.scielo.org.mx/pdf/espiral/v12n34/ v12n34a2.pdf (acceso septiembre 15, 2021).

3 André-Noel Deubel Roth, Politicas públicas: formulación, implementación y evaluación, 13a ed. (Bogotá: Ediciones Aurora, 2018), 206.

4 Ramos y Reyes, "Gobiernos", 52. 
servicios responsivos a los ciudadanos (orientación estratégica, participación al ciudadano, transparencia y acceso a la información)", ${ }^{5}$ pero tales estrategias requieren planificación en los países que las adoptan, los cuales deben involucrar el desarrollo de los supuestos mencionados.

Desde el punto de vista teórico la relación que existe entre NGP local, participación y legitimidad es innegable, debido al aporte de la participación a la construcción de legitimidad de cada Gobierno. Sin embargo, no cualquier tipo de participación ayuda a otorgar valor público: primero, será la participación que vaya más allá del proceso electoral y ponga en práctica nuevos valores como la transparencia, la rendición de cuentas y la equidad; sin los cuales no existe legitimidad social; segundo, la participación por medio de leyes y programas que desenvuelven los postulados constitucionales que reconocen los derechos individuales y colectivos referentes al derecho de participación, y se pone en práctica en acciones concretas para hacerlos explícitos; tercero, el uso de diversas formas de gestión social que permitan "plantear demandas, exigir responsabilidades institucionales; (son) maneras de fomentar la corresponsabilidad pública". ${ }^{6}$

La real vinculación entre la nueva gestión pública y la participación parece reducida en la práctica, ya que para su uso en los escenarios locales se desconoce la complejidad del contexto y se implanta con una rigidez contraria a la descentralización y la autonomía local; no significa que no existen espacios de participación con este modelo, sino que los que existen no entregan a los ciudadanos el poder de decisión que los convierte en actores políticos esenciales. ${ }^{7}$ En el discurso de la NGP se otorga protagonismo al municipio, pues aumenta la capacidad del Gobierno, al recurrir a la participación ciudadana en formulación e implementación de políticas de la ciudad; no obstante, para cumplirlo, se reconoce la necesidad de hacer cambios en la Administración y en los códigos de conducta ética, en especial en los referentes al uso de recursos públicos. ${ }^{8}$ Se trata de nuevos valores y capacidades institucionales y civiles.

\footnotetext{
5 Carlos Gómez Díaz de León, "Nueva gestión pública y gobernanza: desafíos en su implementación", Daena: International Journal of Good Conscience 8, núm. 1 (2013): 183, http://www.spentamexico.org/v8-nl/ A14.8(1)177-194.pdf (acceso septiembre 15, 2021).

$6 \quad$ Ramos y Reyes, "Gobiernos", 54.

7 Diana María Vásquez Avellaneda y Marcela Navarrete Peñuela, "La gobernabilidad local en el contexto de la nueva gestión pública: requisito para a gestión ambiental urbana”, Luna Azul, núm. 39 (2014): 75, http:// www.scielo.org.co/pdf/luaz/n39/n39a05.pdf (acceso septiembre 15, 2021).

8 Vásquez Avellaneda y Navarrete Peñuela, "La gobernabilidad local", 82.
} 


\section{La participación como fuente de legitimidad}

La Carta Iberoamericana de participación ciudadana en la gestión pública, en su Capítulo I, indicó entre los conceptos de la participación ciudadana uno relacionado con los gobiernos, según el cual esta participación les ayuda a "abordar los conflictos y a propiciar acuerdos, así como aumentar la legitimidad y efectividad de las

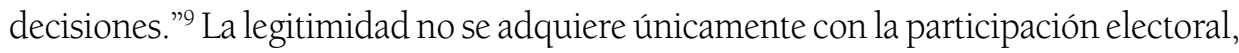
sino cuando tiene lugar en la etapa de diseño y formulación de una política pública.

La participación ciudadana es un espacio que contrapone la democracia participativa y la democracia representativa o las pone en un grado de complementariedad. En indagaciones realizadas en España desde los mismos entes gubernamentales se identifica una nueva valorización de los espacios de participación ciudadana.

En España [...] la mayoría de la población cree precisamente que para mejorar la acción de los políticos se necesita más participación. Y la respuesta institucional y normativa a esta demanda ha sido bastante positiva, de hecho, en la práctica totalidad de los ayuntamientos españoles de tamaño medio (100 000-500 000 habitantes) se han tomado iniciativas participativas, incluso dos Comunidades Autónomas tienen ya direcciones generales de Participación Ciudadana. Obviamente es el ámbito local el más propicio para este tipo de procedimientos. ${ }^{10}$

En el caso de Chile, la participación ciudadana cumple algunas finalidades frente a la gestión. La primera se refiere a la democratización del Estado y del sistema político; la segunda, al mejoramiento de políticas sociales, que es una forma de acceso al Estado, ${ }^{11}$ un factor modernizador de la gestión pública que lleva a comprender un

9 Conferencia Iberoamericana de Ministros de Administración Pública y Reforma del Estado, Carta iberoamericana de participación ciudadana en la gestión pública, Resolución No. 38 del "Plan de Acción de Lisboa" (Lisboa, 25-26 de junio de 2009), 1-15.

10 Manuel Villoria Mendienta, "Las nuevas medidas al servicio de la transparencia, la participación y el control en el gobierno local en la Ley de Suelo", Ciudad y territorio. Estudios territoriales, núm. 26 (2007): 505, https://recyt.fecyt.es/index.php/CyTET/article/download/75787/46199 (acceso septiembre 15, 2021).

11 Álvaro Vicente Ramírez Alujas, "Innovación en la gestión pública y Open Government (gobierno abierto): una vieja nueva idea", Buen Gobierno 1, núm. 1 (2012): 107, https://www.researchgate.net/ publication/228160026_Innovacion_en_la_Gestion_Publica_y_Open_Government_Gobierno_Abierto_ Una_Vieja_Nueva_Idea_Innovation_in_Public_Management_and_Open_Government_An_Old_New_Idea (acceso septiembre 15, 2021). 
nuevo papel del ciudadano, más crítico, activo y reivindicativo. En tercer lugar, la participación ciudadana es un elemento de la descentralización del Estado. ${ }^{12}$

Se ha interpretado este impulso a la participación como una forma de "rescatar la credibilidad perdida por el Estado debido a la crisis del modelo representativo, en un esfuerzo por reconectar con la voluntad popular y legitimar la actividad pública". ${ }^{3}$

La legitimidad es, entonces, una característica que se espera de la gestión pública y también de los escenarios participativos; así, "lo que se requiere son espacios y mecanismos que hagan posible la elaboración deliberativa para concurrir a una decisión a la vez racional, efectiva y legítima". ${ }^{14}$

Se puede hablar de una legitimación interna y una legitimación externa. La legitimación interna hace referencia al mecanismo en sí mismo y sus actores, hasta donde es percibido como legítimo para contribuir a la definición de intereses y prioridades dentro de la agenda pública. En un caso concreto, por ejemplo, el de la participación ciudadana en su forma de control social de la gestión pública, cuando se trata de la manifestación de la voluntad popular de quienes lo realizan, estos deben encontrase legitimados para esta función, lo cual se logra frente a un consenso previo de consulta, discusión y decisión dentro de la organización de control social que determina lo que está autorizado para decir ese vocero en nombre de la organización.

Tener legitimidad para hacer control social significa que la comunidad respalda a su representante porque le cree, tiene confianza en la buena calidad de su trabajo y en su manejo claro y sustentado de la información. La legitimidad se basa, entonces, en la honestidad, transparencia, claridad y respeto con que se actúe. ${ }^{15}$

12 Ramírez Alujas, "Innovación", 108.

13 Andrea Mora Pérez, "Participación, ciudadanía y la nueva gerencia pública en el ámbito local", Heurística: revista digital de historia de la educación, núm. 17 (2014): 208, http://www.saber.ula.ve/bitstream/ handle/123456789/40135/articulo5.pdf?sequence=1\&isAllowed=y (acceso septiembre 15, 2021).

14 Gonzalo Delamaza, "Espacio público y participación ciudadana en la gestión pública en Chile: límites y posibilidades", Revista de la Universidad Bolivariana 10, núm. 30 (2011): 50, DOI: http://dx.doi.org/10.4067/ S0718-65682011000300003 (acceso septiembre 15, 2021).

15 Control Social a la Gestión Pública, "Participación en el control social a la gestión pública: momento de sensibilización", serie Documentos de consulta, módulo I, ed. Comité Académico del Plan Nacional de Formación para el Control Social (Bogotá: Ministerio del Interior y de Justicia, Departamento Administrativo de la Función Pública, 2006), 9. 
La legitimación externa hace referencia al resultado de la deliberación por medio del mecanismo. Muchas veces, la promoción del espacio por parte del Estado se hace con el objetivo de "ser una modalidad para facilitar la adopción de decisiones de impacto social en ámbitos no adecuadamente representados por el sistema político". ${ }^{16}$ Con ello se responde a la pregunta: ¿Cómo puede ser legítima una decisión de gestión si no se toma en consideración el actor principal de las necesidades que busca atender determinada acción? Por la fuente de información utilizada, esta investigación no aborda la legitimación interna, en tanto pretende identificar la participación externa.

Los mecanismos de participación deben contar con la legitimidad vista en ambos sentidos para lograr su propósito. Así lo explica Brugué: "Necesitamos materializar las promesas de participación; unas promesas que no pueden limitarse a la propia realización de los procesos participativos, sino que deben proyectarse sobre su capacidad para mejorar las condiciones de vida de la ciudadanía"; 17 sin embargo, hoy son respaldadas más en su función de legitimación de los dirigentes y de las políticas de Estado. ${ }^{18}$ La legitimidad es, entonces, un resultado que, cuando se busca mediante la participación, debe resultar de un consenso entre actores y fines.

[...] legitimidad y transparencia son la expresión de la confianza entre el ciudadano y la gestión pública local sobre el pacto de una agenda pública consensuada que busca nivelar responsabilidades sobre la base de la inclusión pluralista, la deliberación vinculante en el diseño de las políticas públicas locales, así como el compromiso y la lealtad por lo local en términos de rendición de cuentas. ${ }^{19}$

Hasta aquí, observar la participación ciudadana desde la NGP en su utilidad de construcción de legitimidad implica comprender tal legitimidad en el marco de una serie de valores (transparencia, eficacia, inclusión) y capacidades institucionales y civiles que permite darle más que un uso meramente formal. Además, conlleva comprender la legitimidad no solo en cuanto al resultado de una aceptación o un

16 Delamaza, "Espacio público", 52.

17 Joaquim Brugué, "Una Administración que habla es una Administración que piensa" en Participación ciudadana para una administración deliberativa, coord. Ignacio Celaya Pérez (Zaragoza: Diputación General de Aragón, 2009), 55.

18 Mora Pérez, "Participación", 208.

19 Hilarión Vegas, "Gestión pública local, gobernanza y participación: una visión a partir del discurso de los actores sociales en Venezuela" (Tesis de Doctorado, Universidad de Carabobo, 2015), 278. 
respaldo general, sino en la forma como el respaldo, la convicción o la alineación de valores se materializa.

\section{Metodología}

Analizar el alcance de estos postulados teóricos ha llevado al estudio de un caso concreto de aplicación de la nueva gestión pública local. El referente metodológico que se utilizó fue el documento "Modelo abierto de gestión para resultados", elaborado por el Banco Interamericano de Desarrollo (BID) y el Centro Latinoamericano de Administración para el Desarrollo (CLAD), ${ }^{20}$ a partir del cual se diseñó un instrumento propio que extrae las categorías de análisis referentes a la participación en cada una de las etapas del ciclo de gestión. Dichas categorías se someten a un proceso de adaptación tanto del lenguaje como de la pertinencia de las preguntas respecto a las realidades de la Administración pública de los municipios colombianos.

También se adaptó el instrumento para ser valorado por expertos, de acuerdo con una escala de valor propuesta similar a la del modelo original, que emplea una valoración cuantitativa sobre la información encontrada en las fuentes documentales acerca del ciclo de gestión. Cada ítem sugerido puede ser valorado mediante una escala ordinal de 0 a 5 que ofrece una interpretación tanto cuantitativa como cualitativa. La herramienta aborda cuatro ámbitos de gestión:

Ámbito 1: políticas y estrategias, para "identificar el estado de presencia y desarrollo de los componentes de gestión vinculados a la formulación del programa de gobierno, objetivos, diseño estratégico y organizativo, política económica, presupuesto y acción de gobierno". ${ }^{21}$

Ámbito 2: presupuesto, con el interés de valorar "la presencia y desarrollo de los componentes vinculados a la estrategia económica, del proceso de estructuración del presupuesto, su ejecución y registro contable, los apoyos de la gestión al sistema de información económica y de responsabilidad de la ejecución presupuestaria". 22

Ámbito 3: ejecución, con miras a "identificar el estado de presencia y desarrollo de los componentes del ciclo de gestión vinculados a la ejecución del programa de

\footnotetext{
García y García López, "Modelo abierto", 149.

21 Banco Interamericano de Desarrollo [BID] y Centro Latinoamericano de Administración para el Desarrollo [CLAD], Modelo abierto de gestión para resultados del sector público (Washington: Autor, 2007$), 54$.

22 BID y CLAD, Modelo abierto, 56.
} 
gobierno y de la formulación presupuestaria revisando en profundidad la estructura del ciclo productivo". ${ }^{23}$

Ámbito 4: evaluación, para:

[...] identificar el estado de presencia y desarrollo de los componentes del ciclo de gestión vinculados al proceso de evaluación de la acción de gobierno y de la actuación de la organización pública. Se toman en consideración los sistemas de evaluación de satisfacción de los ciudadanos, métodos de evaluación [...], análisis de resultados estratégicos [...] y métodos e instrumentos para monitorear el cambio social. ${ }^{24}$

En la Tabla 1 se resume la estructura del instrumento.

La valoración de cada calificación se modificó en relación con el instrumento original creado por el BID, según los parámetros que se exponen a continuación:

$0=$ no existe, no se ha planteado o tratado en ningún escenario.

$1=$ se ha tratado, pero no se ha tomado ninguna decisión.

2= se han diseñado actuaciones pendientes de implementación.

3= se han iniciado acciones parciales de implementación.

4= existe un proyecto integral en la fase de implementación.

5= existe un sistema institucional plenamente operativo.

La valoración de cada uno de los componentes fue realizada en forma individual y en dos etapas. La primera fase fue adelantada por tres auxiliares de investigación, quienes organizaron y codificaron la información recopilada, que cumplían el rol de ciudadanos no expertos ocupados en diversos espacios profesionales. La segunda etapa correspondió a la valoración otorgada por cinco expertos, investigadores y docentes de la Universidad Pedagógica y Tecnológica de Colombia (UPTC), la

23 BID y CLAD, Modelo abierto, 58.
24 BID y CLAD, Modelo abierto, 61. 
Universidad Nacional Abierta y a Distancia (UNAD) y la Universidad Antonio Nariño, con sede en el departamento de Boyacá.

La valoración definitiva se obtuvo del promedio de las evaluaciones individuales realizadas por los expertos y los ciudadanos (en la escala de 0 a 5 ), la cual se presenta gráficamente en cada uno de los radares derivados de la tabulación promediada para cada ámbito y los correspondientes componentes del ciclo de gestión.

Para este trabajo, solo se relacionan los hallazgos obtenidos en el conjunto de preguntas asociadas a la participación ciudadana en los municipios analizados. En la adaptación del instrumento se tuvo en cuenta la necesidad de identificar los elementos que más se asociaban con los aspectos que vinculaban la NGP con la participación para la creación de legitimidad local. Uno de los grandes aportes de la utilización de este instrumento es que permitió detectar las cualidades esperadas en las fases del ciclo de gestión de políticas públicas. Así, se han observado aspectos como el valor público de bienes y servicios, la participación en el desarrollo de la misión del Gobierno, la capacidad de adaptación a las circunstancias cambiantes, la presencia de servicios responsivos a los ciudadanos, transparencia, rendición de cuentas y equidad, efectividad de derechos, corresponsabilidad pública, intercambio deliberativo y democratización.

\section{Resultados}

Los municipios analizados han adoptado la NGP en sus actividades administrativas con la forma que ofrece el modelo, pero sin una apropiación directa de sus pretensiones. Ello significa que difícilmente se alcanzarán los propósitos de la participación orientados a equilibrar la introducción de los principios del libre mercado y la empresa en la gestión pública, como muestran los resultados obtenidos. Estos son productos de la evaluación conjunta de los ámbitos de gestión. Desde un punto de vista descriptivo y general, se analizaron las preguntas asociadas con la participación, para luego identificar su calificación acumulada y comparar los tres municipios (Duitama, Sogamoso y Tunja). 
Tabla 1. Categorías asociadas a la participación. Ámbito 1

\begin{tabular}{|c|c|c|}
\hline $\begin{array}{l}\text { Ámbito } 1 . \\
\text { Políticas y }\end{array}$ & \multicolumn{2}{|c|}{ Aspectos decisorios del proceso de formulación de políticas y programas locales } \\
\hline \multirow[t]{3}{*}{ Componentes } & $\begin{array}{l}\text { Información y análisis } \\
\text { económico y social }\end{array}$ & $\begin{array}{l}\text {-Sistemas públicos de información utilizados en la toma } \\
\text { de decisiones. } \\
\text {-Los mecanismos de recolección de la información. } \\
\text {-Su acceso. } \\
\text {-Sostenibilidad. } \\
\text {-Los organismos o las entidades que representan a la } \\
\text { comunidad y su constitución. } \\
\text {-Constitución y divulgación de la propuesta del formula- } \\
\text { dor de la política. }\end{array}$ \\
\hline & $\begin{array}{l}\text { Programas y objetivos de } \\
\text { gobierno }\end{array}$ & $\begin{array}{l}\text {-Participación de grupos sociales en la planeación. } \\
\text {-La integración del consejo territorial de planeación y sus } \\
\text { recomendaciones. }\end{array}$ \\
\hline & $\begin{array}{l}\text { Estrategias básicas de } \\
\text { intervención }\end{array}$ & $\begin{array}{l}\text { - Cooperación público-privada. } \\
\text { - Cooperación con la sociedad civil. }\end{array}$ \\
\hline
\end{tabular}

Fuente: elaboración propia.

Gráfica 1. La participación en el ámbito de políticas y estrategias

\section{ÁMBITO 1. POLÍTICAS Y ESTRATEGİAS}

\section{- CONSOLIDADOS PROMEDIO CALIFICACIÓN FINAL DUITAMA - CONSOLIDADO PROMEDIO CALIFICACIÓN FINAL SOGAMOSO \\ - CONSOlidado PROMEDio Calificación final tUNJa}

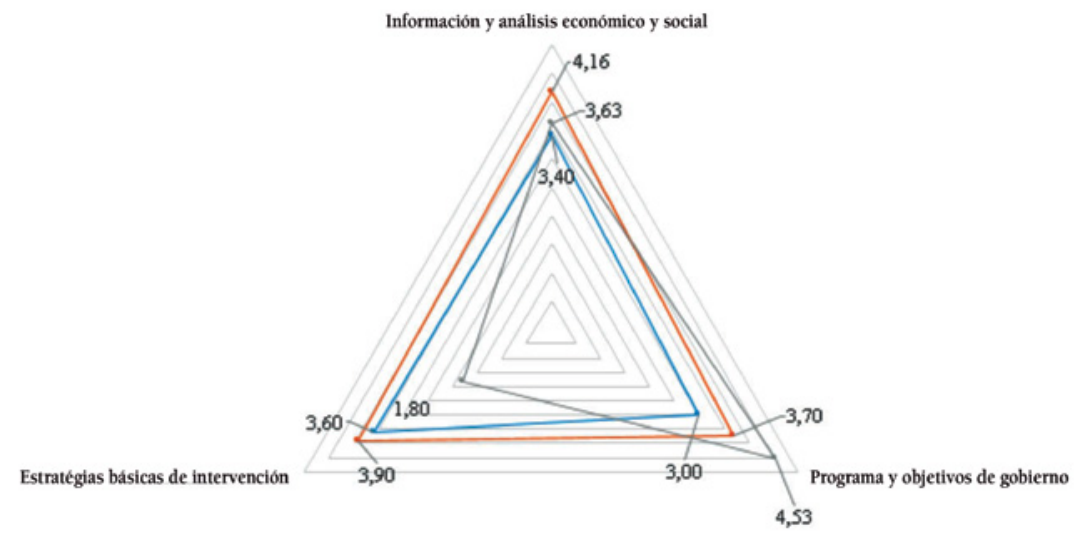

Fuente: elaboración propia.

En este primer ámbito, la calificación se mantuvo entre 3 y 4 puntos, lo que significa que se logró identificar el inicio de acciones parciales de implementación de los aspectos evaluados en esta fase. Sogamoso es la ciudad que más se aproximó a 
tener un proyecto de participación integral con una evaluación de 3,92, es decir, ya en una fase de implementación.

Se observó que los tres municipios dan mayor importancia a la participación en aspectos diversos. Para Duitama, la calificación más alta es 3,60 y la obtuvo en estrategias básicas de intervención, que analiza la cooperación público-privada y la cooperación con la sociedad civil; para Sogamoso, la calificación más alta está en información y análisis económico y social, con 4,16 puntos, en el cual se analizan sistemas públicos de información utilizados en la toma de decisiones y los mecanismos de recolección de la información, su acceso, la sostenibilidad, los organismos o las entidades que representan a la comunidad y su constitución y la divulgación de la propuesta del formulador de la política; por último, Tunja tuvo su mayor fortaleza en ámbitos y objetivos de gobierno, con 4,53, que analizó la participación de grupos sociales en la planeación y la integración del consejo territorial de planeación y sus recomendaciones.

Tabla 2. Categorías asociadas a la participación. Ámbito 2

\begin{tabular}{|c|l|l|}
\hline $\begin{array}{c}\text { Ámbito 2. } \\
\text { Presupuesto }\end{array}$ & \multicolumn{1}{|c|}{$\begin{array}{c}\text { Decisiones económicas en relación con el cumplimiento de las políticas y los } \\
\text { programas municipales }\end{array}$} \\
\hline \multirow{5}{*}{ Componentes } & $\begin{array}{l}\text { Estrategia económica y } \\
\text { financiera }\end{array}$ & $\begin{array}{l}\text {-Posibilidad de auditoría externa sobre los aspectos } \\
\text { financieros. } \\
\text {-La transparencia en la información sobre la deuda. }\end{array}$ \\
\cline { 2 - 3 } & $\begin{array}{l}\text { Procedimiento de ejecución } \\
\text { presupuestario }\end{array}$ & $\begin{array}{l}\text {-Posibilidad de la ejecución del presupuesto con } \\
\text { controles ex ante y ex post. } \\
\text {-Posibilidad de los procedimientos de compras y } \\
\text { contrataciones del municipio. }\end{array}$ \\
\cline { 2 - 3 } & Sistema de seguimiento de la \\
& ejecución y rendición de cuentas & $\begin{array}{l}\text {-Existencia de sistemas estables de información para } \\
\text { el seguimiento de la gestión presupuestaria. } \\
\text {-Posibilidad de auditorías externas anualizadas. } \\
\text {-Análisis de la información. } \\
\text {-Difusión de conclusiones. }\end{array}$ \\
\hline
\end{tabular}

Fuente: elaboración propia. 
Gráfica 2. La participación en el ámbito de presupuesto

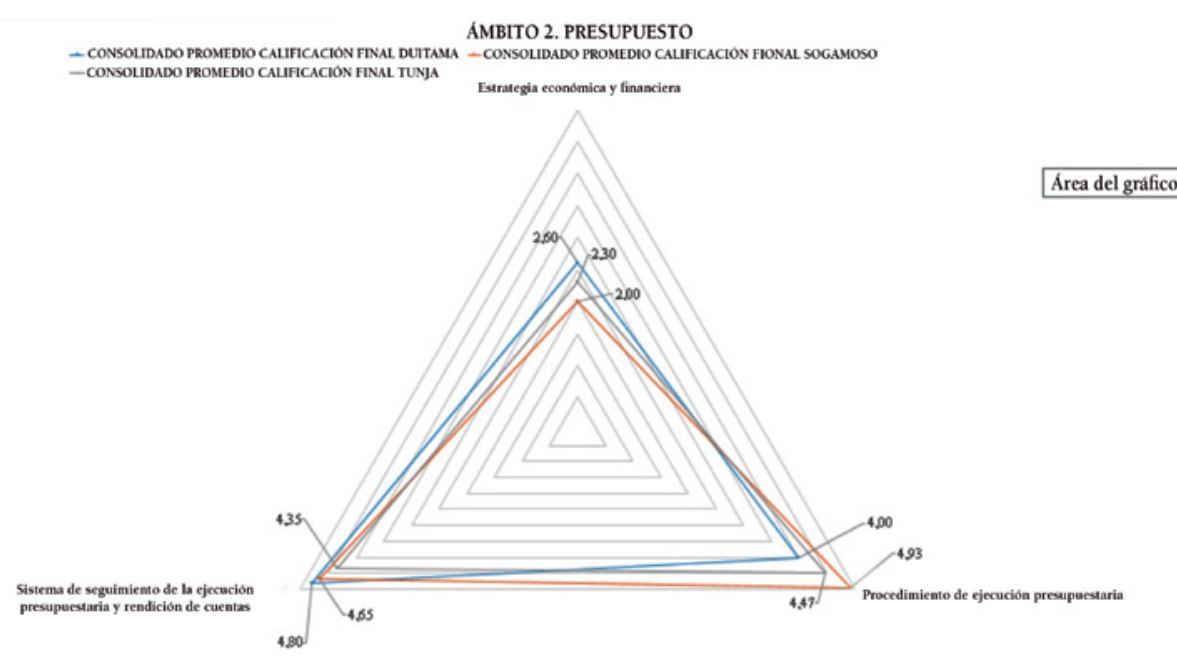

Fuente: elaboración propia.

En el ámbito de presupuesto, la calificación total varió entre 2 y 3,8 puntos. En ningún caso se consolidó un 4 , es decir, un proyecto integral en fase de ejecución en los aspectos referentes a la participación en etapa presupuestal. La mejor calificación la obtuvo la ciudad de Sogamoso, con 3,86 puntos; sin embargo, los tres municipios tuvieron una calificación baja en el uso de espacios de participación en estrategias económicas y financieras, que evaluó elementos como la posibilidad de auditoría externa sobre los aspectos financieros y la transparencia en la información sobre la deuda, en el cual ninguna llegó a los 3 puntos, o sea, apenas se diseñaron acciones, pero estaban pendientes de implementación. En cuanto a las fortalezas, para Duitama, la mayor correspondió al sistema de seguimiento de la ejecución presupuestaria y rendición de cuentas con 4,80 puntos, lo que indica que los participantes de la investigación hallaron un proyecto integral de participación, en fase de implementación; para Sogamoso, la mayor calificación fue en procedimiento y ejecución, en el que se analizó la ejecución del presupuesto con controles ex ante y ex post, así como la posibilidad de los procedimientos de compras y contrataciones del municipio, con una calificación de 4,93; Tunja también tuvo su calificación más alta en este ítem con 4,47 puntos. 
Tabla 3. Categorías asociadas a la participación. Ámbito 3

\begin{tabular}{|c|c|c|}
\hline $\begin{array}{l}\text { Ámbito } 3 . \\
\text { Ejecución }\end{array}$ & \multicolumn{2}{|c|}{$\begin{array}{c}\text { Identificar el estado de presencia y desarrollo de los componentes del ciclo de } \\
\text { gestión vinculados a la ejecución del programa de gobierno y de la formulación } \\
\text { presupuestaria, mediante la revisión en profundidad de la estructura del ciclo } \\
\text { productivo propiamente dicho }\end{array}$} \\
\hline \multirow{4}{*}{ Componentes } & $\begin{array}{l}\text { Estrategias de provisión y } \\
\text { producción }\end{array}$ & $\begin{array}{l}\text {-Disposición de mecanismos para ejercer los derechos de } \\
\text { acceso a bienes y servicios públicos. } \\
\text {-La regulación de la producción privada de bienes y servi- } \\
\text { cios públicos. }\end{array}$ \\
\hline & $\begin{array}{l}\text { Bienes y servicios públicos } \\
\text { en sí mismos }\end{array}$ & $\begin{array}{l}\text {-Disposición de información a los ciudadanos con los } \\
\text { compromisos de la organización sobre los servicios que } \\
\text { ofrece. } \\
\text {-Realización de actividades de I+D para atender más eficaz } \\
\text { y eficientemente a la ciudadanía. }\end{array}$ \\
\hline & $\begin{array}{l}\text { Comunicación de la oferta } \\
\text { pública }\end{array}$ & $\begin{array}{l}\text {-Estrategias de comunicación de la oferta. } \\
\text {-Canales de información de fácil acceso. } \\
\text {-Comunicación orientada a la compresión ciudadana. } \\
\text {-Información exhaustiva. } \\
\text {-Utilización de tecnologías para facilitar el acceso y la cali- } \\
\text { dad de la información. } \\
\text {-Esfuerzos para mantener informados a los sectores } \\
\text { vulnerables. }\end{array}$ \\
\hline & Participación social & $\begin{array}{l}\text {-Existencia de sistemas de participación social que aporten } \\
\text { a la definición de la oferta de servicios. } \\
\text {-Sistemas de participación colectiva o individual para la } \\
\text { identificación de necesidades. } \\
\text {-Mecanismos de colaboración. } \\
\text {-Consulta directa para los planes de mejora. } \\
\text {-Redes público-privadas para la generación de bienes } \\
\text { públicos. } \\
\text {-Evidencia de participación ciudadana en acuerdos } \\
\text { municipales. } \\
\text {-Existencia de juntas administradoras locales. } \\
\text {-Participación de ciudadanos en funciones de control y } \\
\text { vigilancia. }\end{array}$ \\
\hline
\end{tabular}

Fuente: elaboración propia. 
Gráfica 3. La participación en el ámbito de ejecución

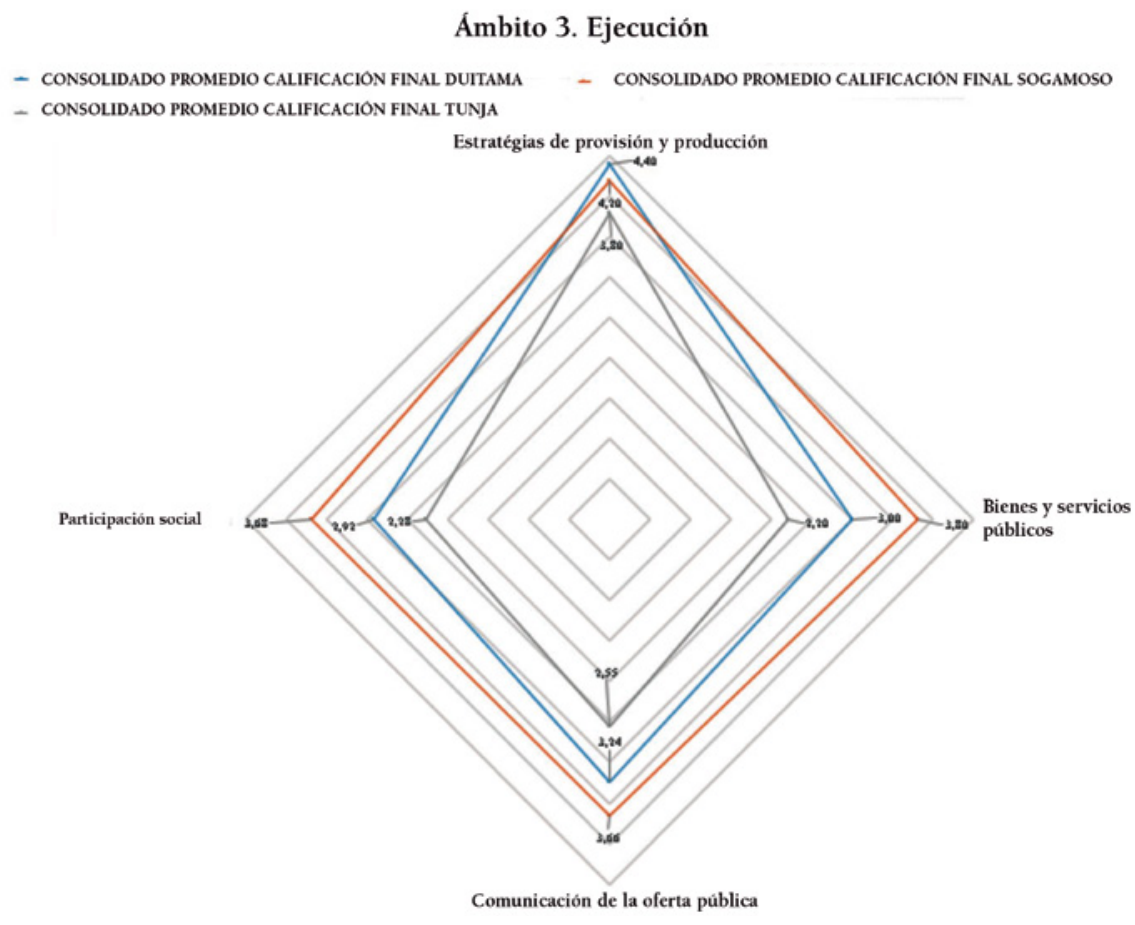

Fuente: elaboración propia.

En este ámbito, el mayor rezago lo presentó Tunja, cuyo promedio acumulado fue 2,71, es decir, apenas se han diseñado acciones, pero estaban en proceso de implementación. El mejor desempeño general lo obtuvo la ciudad de Sogamoso, que alcanzó 3,83 puntos.

En los aspectos específicos, los resultados fueron muy similares en los tres municipios. La calificación más alta correspondió a estrategias de provisión y producción, con 4,40 puntos para Duitama, 4,20 para Sogamoso y 3,80 para Tunja. Los evaluadores estimaron que ya existía un proyecto integral en fase de implementación en elementos como la disposición de mecanismos para ejercer los derechos de acceso a bienes y servicios públicos y la regulación de la producción privada de bienes y servicios públicos.

La calificación más baja fue en participación social: Duitama obtuvo 2,92 puntos; Sogamoso, 3,68 —aunque fue más baja en comunicación de oferta pública con 3,66- y Tunja, 2,28; esto significa que apenas se identificó el diseño de acciones 
que, en su mayoría, estaban pendientes por implementar. Este aspecto evalúa la existencia de sistemas de participación social que definan la oferta de servicios, los sistemas de participación colectiva o individual para detectar necesidades, mecanismos de colaboración, consulta directa para los planes de mejora, relación estable con la sociedad civil, regulaciones sobre las consultas públicas, redes público-privadas para generar bienes públicos, evidencia de participación ciudadana en acuerdos municipales, existencia de juntas administradoras locales y participación de ciudadanos en funciones de control y vigilancia.

Tabla 4. Categorías asociadas a la participación. Ámbito 4

\begin{tabular}{|c|c|c|}
\hline Ámbito 4. & \multicolumn{2}{|c|}{ Evaluar la acción del Gobierno y de la organización pública } \\
\hline \multirow{4}{*}{ Componentes } & $\begin{array}{l}\text { Evaluación de la producción y } \\
\text { asignación de valor público }\end{array}$ & $\begin{array}{l}\text {-Evaluación del proceso de entrega de bienes a la } \\
\text { ciudadanía. } \\
\text {-Análisis cualitativo de esas evaluaciones. }\end{array}$ \\
\hline & Análisis de satisfacción & $\begin{array}{l}\text {-Existencia de mecanismos para evaluar el grado de } \\
\text { satisfacción de los ciudadanos. } \\
\text {-Regularidad de estos sistemas y su comparación } \\
\text { temporal. } \\
\text {-Desagregación territorial y social de los resultados de } \\
\text { satisfacción. } \\
\text {-Existencia de un sistema transparente de quejas y } \\
\text { atención a las quejas de la ciudadanía en los planes de } \\
\text { mejora. }\end{array}$ \\
\hline & $\begin{array}{l}\text { Resultados estratégicos y de } \\
\text { impacto y cambios interme- } \\
\text { dios de la acción del Gobierno }\end{array}$ & $\begin{array}{l}\text {-Análisis de cambios sociales producidos por el des- } \\
\text { ajuste del resultado. } \\
\text {-Evaluación articulada de procesos de planeación y } \\
\text { presupuestación. }\end{array}$ \\
\hline & Rendición de cuentas & $\begin{array}{l}\text {-Uso de indicadores adecuados. } \\
\text {-Sistemas estadísticos fiables. } \\
\text {-Información estadística homologable para todo el país. } \\
\text {-Publicidad de la evaluación ad hoc sobre la acción } \\
\text { institucional. } \\
\text {-Rendición de cuentas a la ciudadanía sobre los resulta- } \\
\text { dos de la gestión. } \\
\end{array}$ \\
\hline & $\begin{array}{l}\text { Participación y utilización de } \\
\text { evaluación }\end{array}$ & $\begin{array}{l}\text {-Accesibilidad de las evaluaciones a la organización. } \\
\text {-Utilización de la evaluación en la toma de decisiones. } \\
\text {-Evaluación en la base del rediseño de los programas de } \\
\text { gobierno. } \\
\text {-Discusión pública de los resultados de las evaluaciones. }\end{array}$ \\
\hline & Análisis de la realidad social & $\begin{array}{l}\text {-Sistemas de información para evaluar las demandas y } \\
\text { necesidades sociales y conocer los grados de cobertura } \\
\text { de la acción institucional sobre estas demandas. }\end{array}$ \\
\hline
\end{tabular}

Fuente: elaboración propia. 
Gráfica 4. Participación en el ámbito de evaluación

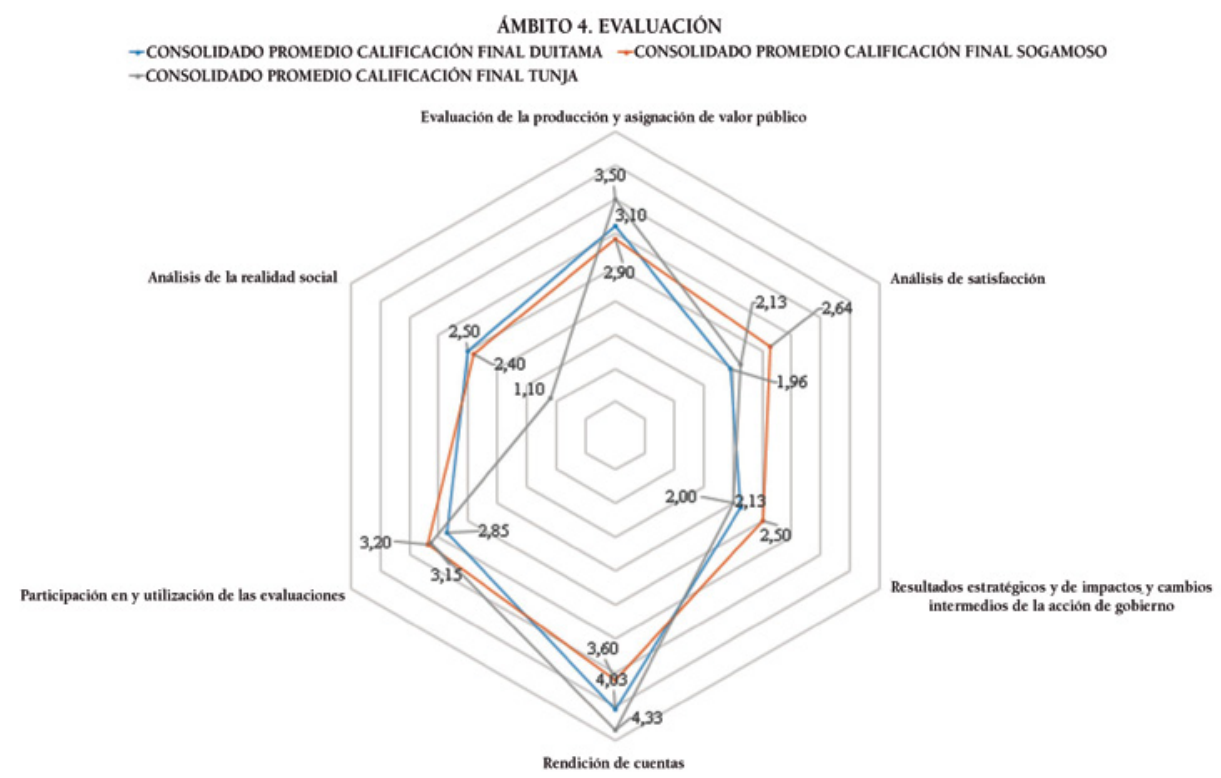

Fuente: elaboración propia.

El último ámbito estudiado es el que obtuvo menores calificaciones generales. La participación en la etapa de evaluación de la gestión de los tres municipios osciló entre 2,70 y 2,87: nuevamente se diseñaron acciones, pero quedaron pendientes de ser implementadas. En los aspectos específicos, las tres ciudades obtuvieron su mejor calificación en rendición de cuentas, que contempló el uso de indicadores adecuados, sistemas estadísticos fiables, información estadística homologable a todo el país, publicidad de las evaluaciones ad hoc sobre la acción institucional, rendición de cuentas a la ciudadanía sobre los resultados de la gestión. Duitama tuvo 4,03; Sogamoso, 3,60, y Tunja, 4,33.

Las menores calificaciones se obtuvieron así: Duitama, en análisis de satisfacción, con una calificación de 1,96 , es decir, son puntos que se trataron, pero no se tomó una decisión en cuanto a existencia de mecanismos para evaluar el grado satisfacción de los ciudadanos, regularidad de estos sistemas y su comparación temporal, desagregación territorial y social de los resultados de satisfacción, existencia de un modelo transparente de quejas y atención a las quejas de la ciudadanía en los planes de mejora. Para Sogamoso correspondió al análisis de la realidad social, con 2,40, al igual que para la ciudad de Tunja, con 1,10, que revisó si los sistemas de 
información permitían evaluar las demandas y necesidades sociales y conocer la cobertura de la acción institucional sobre estas demandas.

\section{Conclusiones}

La metodología de análisis permite cuestionar hasta qué punto los Gobiernos locales examinados incorporan la participación a sus gestiones, para así materializar los valores de la nueva gestión pública y que ya fueron enunciados, como el incremento de valor público de los bienes y servicios, diálogo, participación en el desarrollo de las misiones del Gobierno, servicios responsivos a los ciudadanos, transparencia, rendición de cuentas, equidad, existencia de acciones concretas para hacer explícitos espacios que concedan derechos individuales y colectivos, formas de gestión social que faciliten plantear demandas y exigir responsabilidades y corresponsabilidad pública, intercambio deliberativo y decisorio para la construcción de políticas y programas, transparencia, confianza legítima y democratización del Estado; todos ellos como partes fundamentales de la construcción de legitimidad del Gobierno.

Observar la participación en el primer ámbito de la fase inicial del ciclo de gestión de políticas públicas y programas en los tres municipios seleccionados permite dar cuenta del uso de aquella en la misión y concreción del Gobierno, el incremento del valor público, la posibilidad de plantear demandas y el intercambio deliberativo. Como se vio en los resultados, es dable concluir que en esta etapa se está en un proceso de interiorización, lo cual se refleja en que los tres municipios iniciaron acciones parciales de participación; sin embargo, esta fue en una sola vía, mediante la entrega de información de la Administración a la comunidad y garantizar su representación, sin que esto implicara su vinculación a la elaboración de los objetivos del plan y las acciones a realizar y mucho menos el registro de sus aportes, aunque no es una situación explícitamente exigida por la NGP.

El ámbito número dos, que analiza el presupuesto, muestra la actuación de la Administración entre las etapas de formulación y ejecución de políticas públicas y programas. Aquí se observa la concreción de valores como la transparencia y la existencia de acciones concretas que haga explícitos los espacios de participación. Los tres municipios iniciaron acciones parciales de implementación, aunque en las estrategias económicas y financieras obtuvieron una baja calificación en la posibilidad de auditorías externas sobre los aspectos financieros y la transparencia en la información sobre la deuda, así que no se encontraron elementos significativos 
que reflejaran la preocupación de las Administraciones por la participación, lo que afecta directamente la construcción de una confianza legítima.

El tercer ámbito, llamado ejecución, se concentra en esta etapa del ciclo. Se examina el grado de concreción de cualidades o valores como la creación de valor público, acciones concretas para hacer explícitos los espacios de participación, transparencia, intercambio deliberativo y diversidad de formas de gestión social que permitan a los ciudadanos plantear demandas y exigir responsabilidades. Se halló que en los municipios se iniciaron acciones parciales de implementación, pero es lamentablemente el ámbito con el más bajo uso de la participación en su componente de participación social, en el cual apenas se diseñaron acciones pendientes de implementación. Es aquí donde se comienza a notar la baja prioridad y la poca voluntad política para abrir un espacio significativo a la participación en la ejecución de políticas y planes de gobierno.

El cuarto ámbito, que analiza la fase de evaluación, expone si se ha generado valor público, adaptación de las Administraciones a las circunstancias cambiantes, democratización del Estado, rendición de cuentas, diversidad de formas de gestión social que permitan a los ciudadanos plantear demandas y exigir responsabilidades, intercambio deliberativo y decisivo, y transparencia. Este arrojó la más baja calificación, ya que se diseñaron acciones, pero estaban pendientes de implementación; esto no es extraño, dado que esta etapa es la más relegada en términos de estudios académicos y prácticos. Se puede colegir que a los Gobiernos locales estudiados no les interesó ser evaluados por la ciudadanía ni conocer la efectividad de sus acciones; de esta manera, la participación no logró un papel deliberativo y decisorio. Paradójicamente, el aspecto mejor evaluado aquí fue la rendición de cuentas, así que, de nuevo, la participación fue en una sola vía: las Administraciones entregaron información, pero no les concierne la retroalimentación ciudadana.

Como se dijo, con este artículo no se pretendía analizar la legitimidad interna de la participación en un panorama de Nueva Gestión Pública local, sino encontrar indicios de la legitimidad externa. Es claro que la participación tiene mayor importancia para los Gobiernos locales en las primeras etapas del ciclo de gestión de política pública, porque es allí donde la legitimidad adquiere un mayor peso para el mantenimiento de la estabilidad política, pero en la ejecución y la evaluación no dan mayor espacio a la participación ciudadana; luego, se infiere un menor interés por consolidar mayor legitimidad sobre su gestión, lo que lleva a concluir 
que la interiorización de los valores de una buena participación están lejos de ser adoptados sustancialmente y no son sino aspectos formales de su enunciación.

\section{Referencias}

Banco Interamericano de Desarrollo [BID] y Centro Latinoamericano de Administración para el Desarrollo [CLAD]. Modelo abierto de gestión para resultados del sector público. Washington: Autor, 2007.

Brugué, Joaquim. "Una Administración que habla es una Administración que piensa" en Participación ciudadana para una Administración deliberativa, coordinado por Ignacio Celaya Pérez, 55-72. Zaragoza: Diputación General de Aragón, 2009.

Conferencia Iberoamericana de Ministros de Administración Pública y Reforma del Estado. Carta iberoamericana de participación ciudadana en la gestión pública, Resolución No. 38 del "Plan de Acción de Lisboa". Lisboa, 25-26 de junio de 2009.

Control Social a la Gestión Pública. "Participación en el control social a la gestión pública: momento de sensibilización", serie Documentos de consulta, módulo I, editado por Comité Académico del Plan Nacional de Formación para el Control Social. Bogotá: Ministerio del Interior y de Justicia, Departamento Administrativo de la Función Pública, 2006.

Delamaza, Gonzalo. "Espacio público y participación ciudadana en la gestión pública en Chile: límites y posibilidades". Revista de la Universidad Bolivariana 10, núm. 30 (2011): 45-75, DOI: http://dx.doi.org/10.4067/S0718-65682011000300003 (acceso septiembre 15, 2021).

Díaz de León, Carlos Gómez. "Nueva gestión pública y gobernanza: desafíos en su implementación". Daena: International Journal of Good Conscience 8, núm. 1 (2013): 177-194, http://www.spentamexico.org/v8-n1/A14.8(1)177-194.pdf (acceso septiembre 15, 2021).

García, Mauricio y Roberto García López. "Modelo abierto de gestión para resultados en el sector público". Revista del CLAD Reforma y Democracia, núm. 39 (2007): 149-210, https://www.redalyc.org/pdf/3575/357533693007.pdf (acceso septiembre 15, 2021).

Mora Pérez, Andrea. "Participación, ciudadanía y la nueva gerencia pública en el ámbito local". Heuristica: revista digital de historia de la educación, núm. 17 (2014): 207-215, http://www. saber.ula.ve/bitstream/handle/123456789/40135/articulo5.pdf?sequence=1\&isAllowed $=y$ (acceso septiembre 15, 2021).

Ramírez Alujas, Álvaro Vicente. "Innovación en la gestión pública y Open Government (gobierno abierto): una vieja nueva idea”. Buen Gobierno 1, núm. 1 (2012): 96-132, https://www.researchgate.net/publication/228160026_Innovacion_en_la_Gestion_Publica_y_Open_Government_Gobierno_Abierto_Una_Vieja_Nueva_Idea_Inno- 
vation_in_Public_Management_and_Open_Government_An_Old_New_Idea (acceso septiembre 15, 2021).

Ramos, José María y Marcela Reyes. "Gobiernos locales y participación ciudadana: hacia un enfoque de gestión estratégica asociada”. Espiral 12, núm. 34 (2005): 39-66, http:// www.scielo.org.mx/pdf/espiral/v12n34/v12n34a2.pdf (acceso septiembre 15, 2021).

Roth Deubel, André-Noel. Politicas públicas: formulación, implementación y evaluación. 13a ed. Bogotá: Aurora, 2018.

Vásquez Avellaneda, Diana María y Marcela Navarrete Peñuela. "La gobernabilidad local en el contexto de la nueva gestión pública: requisito para la gestión ambiental urbana". Luna Azul, núm. 39 (2014): 63-88, http://www.scielo.org.co/pdf/luaz/n39/n39a05. pdf (acceso septiembre 15, 2021).

Vegas, Hilarión. "Gestión pública local, gobernanza y participación: una visión a partir del discurso de los actores sociales en Venezuela". Tesis de Doctorado, Universidad de Carabobo, 2015.

Villoria Mendienta, Manuel. "Las nuevas medidas al servicio de la transparencia, la participación y el control en el gobierno local en la Ley de Suelo". Ciudad y territorio. Estudios territoriales, núm. 26 (2007): 493-513, https://recyt.fecyt.es/index.php/ CyTET/article/download/75787/46199 (acceso septiembre 15, 2021). 